\title{
Role of Pathology and Immunohistochemistry in the New Era Of Molecular Therapy
}

\author{
Atif Ali Ahmed*
}

Children's Mercy Hospital-University of Missouri, Kansas City, Missouri, USA

\begin{abstract}
The modern practice of pathology entails increasing involvement with management of cancer patients. Pathologists are often faced with clinical and research involvements that obligate them to search for prognostic and therapeutic markers on patients' tumor samples. A practical example would be the detection of HER2-neu in breast cancer which has revolutionized the use of prognostic-therapeutic immunohistochemistry (IHC) in the practical management of patients. Numerous other markers and proliferation pathways have recently been elucidated in many human cancers including pediatric cancers. Although most of these markers are being detected by conventional molecular techniques, IHC methods, routinely practiced in histopathologic laboratories, are being employed at an increasing pace. Since most of these proliferation markers are also the targets of new specific therapy, their positive identification often correlates with the appropriateness of that specific therapy and with other parameters that can gauge the patient's prognosis. A good example is the identification of epidermal growth factor receptor by IHC in the patient's lung tumor as an indicator of the tumor responsiveness to "Gefitinib". Such potential application of immunohistochemistry in patients' care necessitates adequate standardization of this technique to optimize patients' management, since erroneous results can lead to erroneous management of patients with cancer. These facts will initiate a new role for pathology laboratory departments to provide a stronger impact on patients' managements.
\end{abstract}

Keywords: Immunohistochemistry, biomarkers, cancer.

\section{INTRODUCTION}

The past decades have witnessed significant improvements in pathology and laboratory medicine as they apply to diagnosis and management of patients. New research developments have contributed to increased understanding of pathogenesis of various neoplastic and nonneoplastic diseases. Immunohistochemistry and molecular pathology are two major ancillary diagnostic services that were established and evolved as direct impact of major research findings. These research developments are incorporated into diagnostic laboratories and have resulted in parallel increase in number and complexity of tests offered in diagnostic pathology. Many of these developments and new tests were in the area of cancer biology. Proliferation pathways in numerous cancers have been delineated and characterized. Member molecules of these pathways have been identified and their expression patterns serve as indicators or biomarkers of specific state of carcinogenesis. These biomarkers and their molecular alterations can now be detected in clinical labs by routine methods [1].

\section{NEW CANCER MARKERS AND THEIR DETECTION}

New anticancer drugs target or inhibit these specific proteins or biomarkers and their molecular alterations present in cancer cells. Pathology role in patients' managements depends on identification of the expression of biomarkers in tissue specimens from patients with cancer or

*Address correspondence to this author at the Children's Mercy HospitalUniversity of Missouri, Kansas City, Missouri, USA; Tel: 816-234-3234: Fax: 816-802-1492; E-mail: aahmed@cmh.edu other lesions. Identification of biomarkers may enable selection of patients most likely to respond to molecularly targeted drugs, allow real-time monitoring of treatment efficacy or identify early signs of toxicity and facilitate decision making during clinical evaluation and start of therapy. Biomarker expression in tumorous tissue taken from patients can de detected by immunohistochemistry and/or molecular pathology. Identification of biomarkers in pathology can be usefully employed in different ways: 1) as diagnostic markers: e.g. immunohistochemical expression of C-kit protein in gastrointestinal stromal tumors, 2) as prognostic markers that can independently forecast clinical outcome, and as 3) predictive markers that can independently predict response to a particular therapy [2,3]. An example of such use would be the identification of estrogen receptors. Estrogen receptors in breast cancer are strong predictive markers for response to tamoxifen therapy.

Targeted signal transduction or proliferation pathways in cancers can be classified in many ways [4], most commonly as follows:

- Receptor kinases:

a. Epidermal growth factor receptor

b. Her2/neu

c. C-kit

- Hepatocyte growth factor/ C-met pathway

- Insulin growth factor receptor pathway

- Intracellular signaling kinases:

a. Src 


\section{b. PI3k/Akt/mTOR pathway}

c. Mitogen-activated Protein Kinase pathway: Ras, Raf, MEK

- Angiogenesis pathways

- Integrins and extracellular matrix proteins

- Heat shock protein

- Ubiquitin-proteosome system

- Apoptosis enhancers: TRAIL, survivin

- Mitotic Kinases: Aurora, kinesin spindle protein

- DNA-RNA enzymes: DNA methyltransferase, Histone deacetylases

- poly(ADP-ribose) polymerase

\section{BIOMARKERS IN PATHOLOGY}

Many of these proteins can serve as biomarkers for certain cancers and be detected in hospital pathology laboratories in more than one particular method. These methods include non-morphologic methods applied in molecular pathology laboratories or morphologic methods that can be visualized on tissue sections such as fluorescent in situ hybridization (FISH), chromogenic in situ hybridization (CISH) and Immunohistochemistry (IHC). Morphologic methods are more optimal, widely available and easy to perform. Biomarkers of proven diagnostic value in pathology and patient benefit include:

\section{Estrogen Receptors in Breast Cancer}

It has been long established that the presence of estrogen receptors in breast cancer tissue demonstrable by routine IHC is an indication for tamoxifen therapy. Today immunohistochemical detection of estrogen and progesterone receptors is part of the routine work-up of breast cancer [5].

\section{Her2/neu}

This marker is identified in $10-20 \%$ of breast cancer patients. Breast cancer patients with positive expression have a significantly shorter disease-free survival and overall survival. Breast cancers with Her2/neu alterations (detected by IHC or FISH) are critical to identify because such tumors require targeted therapies such as trastuzumab. Although Her2/neu can be detected by numerous methods, only two methods are currently approved and recommended for its detection: IHC and FISH. Standardized immunohistochemical techniques exhibit a very good correlation with FISH methods. Such standardization requires the use of $10 \%$ neutral-buffered formalin as a fixative allowing at least 6-8 hours of tissue fixation, not $>48 \mathrm{hrs}$. Interpretation of IHC results also requires a scoring system which is developed by the American society of clinical oncology and the college of American pathologists [5-7].

\section{C-kit/PDGFR}

Mutations have been detected and targeted in malignant gastrointestinal stromal tumors, chronic myeloid leukemia, and dermatofibrosarcoma protuberans. Activating C-kit mutations are also described in other tumors including seminomas, mastocytosis, leukemias, and melanomas, suggesting a broader role in oncogenesis. These markers serve as therapeutic targets of imatinib mesylate and sunitinib and indication of therapy depends on identification of specific c-kit or PDFGR mutations. Protein expression by IHC may be useful for the diagnosis but does not detect genetic mutations [8].

\section{BRAF}

Mutations have been detected in melanomas, papillary thyroid cancer, colorectal carcinomas and less frequently also in other solid epithelial tumors. Mutations have recently been detected in pediatric gliomas but are less frequent in sarcomas. Mutations, such asV600E mutation, are commonly detected by molecular techniques and IHC methods are less helpful. It has recently been identified that BRAF mutations can serve as marker of tumor response to therapy. Patients with metastatic colorectal cancer resistant to cetuximab and panitumumab are found to have KRAS or BRAF mutations. Treatment with the BRAF inhibitor "sorafenib" restored sensitivity to panitumumab or cetuximab of colorectal cancer cells with V600E allele mutations [9].

\section{KRAS}

KRAS mutations have been identified in cancers of the pancreas (90\%), colon (50\%), lung (30\%) and thyroid (50\%), acute leukemia (5-30\%) and chronic myelomonocytic luekemias $(65 \%)$. Similar to BRAF mutations, KRAS can serve as prognostic marker for colon cancer patients treated with epidermal growth factor receptor-targeted therapies [1012]. KARS mutations are commonly detected in molecular pathology labs and immunostains are less helpful.

\section{Epidermal growth factor receptor (EGFR)}

This receptor plays a significant role in lung and colon carcinogenesis. High expression is observed in squamous cell carcinomas, large cell and adenocarcinomas and bronchial preneoplastic lesions. High EGFR expression is found to be an adverse prognostic factor in non-small cell lung carcinoma (NSCLC) [13]. EGFR expression can be determined by IHC and/or FISH with conflicting study results and lack of excellent correlation between the two methods. EGFR expression as detected by IHC is not an effective predictor of response to gefitinib. Immunohistochemical detection of EGFR in paraffin-embedded tumor tissues varies in staining intensity due to choice of fixative and storage time of tissue sections. Frequency of detecting EGFR expression may also vary on the choice of antibody manufacturer. Dako antibody provided the best discrimination between EGFR-positive and EGFR-negative patients. In spite of that, demonstration of positive expression by IHC or FISH is often required before starting therapy with gefitinib or erlotinib in lung or colon cancer patients [14-16].

\section{Insulin-Like Growth Factor (IGF) Receptor Family Pathway}

This family consists of IGF-1, IGF-2, and IGF-1R. Several IGF-1R inhibitors are available with broad clinical anti-tumor activity. Various members of this pathway are expressed in non-small lung cancer, breast, colorectal, Hodgkin's lymphoma, sarcomas, ovarian, and pancreatic malignancies. IGF-1R is frequently activated in lung cancer, 
plays a role in NSCLC tumorigenesis and progression and is associated with poor patients' prognosis [17]. Both FISH methods and immunostains are practical and helpful.

\section{Angiogenesis Markers}

Vascular endothelial growth factor (VEGF) is the prototype of this family that includes more than one member and their receptors. Numerous studies have shown that immunohistochemical expression of VEGF and receptors can serve as predictive markers for several cancers. Positive VEGF immunostaining independently predicts poor prognosis in gastric cancer patients. Strong VEGF staining showed a significant correlation with both short time of relapse and short survival in NSCLC [18]. Increased expression is also a prognostic marker in papillary thyroid carcinoma. In colorectal carcinoma, VEGF Correlates with advanced stage and poor prognosis.

Research is still being conducted on other biomarkers to determine their potential diagnostic usefulness in pathology [4]. These biomarkers have more ubiquitous distribution in many cancers and their detection in pathology laboratories may not be of value as targeted therapy can be initiated as soon as the diagnosis is made. Examples of these would be:

Src

This is a non receptor protein kinase that is dysregulated in several human cancers, including breast, colorectal, lung and ovarian and hematological malignancies. Immunostains are available for research use.

\section{P13/Akt/mTOR Pathways}

This is a ubiquitous pathway, becoming increasingly identified in numerous cancers. Immunostains are available for research use. Studies in different tumors are still being undertaken to determine the percentage of tumors with positive expression and correlate the expression with patient's prognostic parameters and response to treatment.

\section{NF-Kappa B}

This molecule is widely available in numerous cancers and is targeted by proteosome inhibitors.

\section{STANDARDIZATION OF IMMUNOHISTOCHEM- ICAL METHODS}

It is clear that the role of immunohistochemistry in detecting biomarker expression in pathology depends largely on research studies that demonstrate differential immunohistochemical expression and other studies that show good correlation between positive expression and response to new therapy. Once the potential value of a new immunohistochemical test method is appreciated, the burden will be to ensure the standardization of the testing protocol to maintain conformity of the test and minimize interlaboratory variation. Research studies performed on Her2/neu and EGFR can not underemphasize the importance of test standardization in immunohistochemistry. Results may vary widely depending on the choice of fixative, choice of antibody manufacturer, and the type of immunostaining methods. A scoring system of test results should be regularly adopted and properly reported. In the event of equivocal results, a back-up test method by another technique such as molecular or FISH methods should be made available. Once these parameters are standardized, immunohistochemistry will assume a better and well-defined role in management of patients with cancer.

\section{REFERENCES}

[1] Kreeger, P.K.; Lauffenburger, D.A. Cancer systems biology: a network modeling perspective. Carcinogenesis 2010, 31, 2-8.

[2] Cummings, J.; Ward, T.H.; Greystoke, A.; Ranson, M.; Dive, C. Biomarker method validation in anticancer drug development. $\mathrm{Br}$. J. Pharmacol., 2008, 153, 646-656.

[3] MacConaill, L.E.; Campbell, D.C.; Kehoe, S.M.; Bass, A.J.; Hatton, C.; Niu, L.; Davis, M.; Yao, K.; Hanna, M.; Mondal, C.; Luongo, L.; Emery, C.M.; Baker, A.C.; Philips, J.; Goff, D.J.; Fiorentino, M.; Rubin, M.A.; Polyak, K.; Chan, J.; Wang, Y.; Fletcher, J.A.; Santagata, S.; Corso, G.; Roviello, F.; Shivdasani, R.; Kieran, M.W.; Ligon, K.L.; Stiles, C.D.; Hahn, W.C.; Meyerson, M.L.; Garraway, L.A. Profiling critical cancer gene mutations in clinical tumor samples. PLoS One, 2009, 4, 1-7.

[4] Ma, W.W.; Adjei, A.A. Novel agents on the horizon for cancer therapy. CA. Cancer J. Clin., 2009, 59, 111-137.

[5] Gown, A.M. Current issues in ER and HER2 testing by IHC in breast cancer. Mod. Pathol., 2008, 21, S8-S15.

[6] Hicks, D.G.; Kulkarni, S. HER2+ breast cancer review of biologic relevance and optimal use of diagnostic tools. Am. J. Clin. Pathol., 2008, 129, 263-273.

[7] Oyama, T.; Ishikawa, Y.; Hayashi, M.; Arihiro, K.; Horiguchi, J. The effects of fixation, processing and evaluation criteria on immunohistochemical detection of hormone receptors in breast cancer. Breast Cancer, 2007, 14, 182-188.

[8] Badalamenti, G.; Rodolico, V.; Fulfaro, F.; Cascio, S.; Cipolla, C.; Cicero, G.; Incorvaia, L.; Sanfilippo, M.; Intrivici, C.; Sandonato, L.; Pantuso, G.; Latteri, M. A.; Gebbia, N.; Russo, A. Gastrointestinal stromal tumors (GISTs): focus on histopathological diagnosis and biomolecular features. Ann. Oncol., 2007, 18 (Suppl 6), vi136-vi140.

[9] Di Nicolantonio, F.; Martini, M.; Molinari, F.; Sartore-Bianchi, A.; Arena, S.; Saletti, P.; De Dosso, S.; Mazzucchelli, L.; Frattini, M.; Siena, S.; Bardelli, A. Wild-type BRAF is required for response to panitumumab or cetuximab in metastatic colorectal cancer. J. Clin. Oncol., 2008, 26, 5705-12.

[10] Wang, H.L.; Lopategui, J.; Amin, M.B.; Patterson, S.D. KRAS mutation testing in human cancers: The pathologist's role in the era of personalized medicine. Adv. Anat. Pathol., 2010, 17, 23-32.

[11] Siena, S.; Sartore-Bianchi, A.; Di Nicolantonio, F.; Balfour, J.; Bardelli, A. Biomarkers predicting clinical outcome of epidermal growth factor receptor-targeted therapy in metastatic colorectal cancer. J. Natl. Cancer Inst., 2009, 101, 1308-24.

[12] Hamilton, S.R. Targeted therapy of cancer: new roles for pathologists in colorectal cancer. Mod. Pathol., 2008, 21, S23-S30.

[13] Zhu, C.Q.; Shih, W.; Ling, C.H.; Tsao, M.S. Immunohistochemical markers of prognosis in non-small cell lung cancer: a review and proposal for a multiphase approach to marker evaluation. J. Clin. Pathol., 2006, 59, 790-800.

[14] Dziadziuszko, R.; Hirsch, F.R.; Varella-Garcia, M.; Bunn, P.A. Selecting lung cancer patients for treatment with epidermal growth factor receptor tyrosine kinase inhibitors by immunohistochemistry and fluorescence in situ hybridization - why, when, and how? Clin. Cancer Res., 2006, 12 (14 Suppl), 4409s-4415s.

[15] Atkins, D.; Reiffen, K.A.; Tegtmeier, C.L.; Winther, H.; Bonato, M.S.; Störkel, S. Immunohistochemical Detection of EGFR in paraffin-embedded tumor tissues: Variation in staining intensity due to choice of fixative and storage time of tissue sections. $J$. Histochem. Cytochem., 2004, 52, 893-901.

[16] Hirsch, F.R.; Dziadziuszko, R.; Thatcher, N.; Mann, H.; Watkins, C.; Parums, D.V.; Speake, G.; Holloway, B.; Bunn, P.A.; Franklin, W.A. Epidermal growth factor receptor immunohistochemistry. Comparison of antibodies and cutoff points to predict benefit from 
gefitinib in a phase 3 placebo-controlled study in advanced nonsmall-cell lung cancer. Cancer, 2008, 112, 1114-1121.

[17] Yin, M.; Guan, X.; Liao, Z.; Wei, Q. Insulin-like growth factor-1 receptor-targeted therapy for non-small cell lung cancer: a mini review. Am. J. Transl. Res., 2009, 1, 101-114.

[18] Carrillo-de Santa Pau, E.; Arias, F.C.; Caso-Peláez, E.; MuñozMolina, G.M.; Sánchez-Hernández, I.; Muguruza-Trueba, I.;
Moreno-Balsalobre, R.; Sacristán-López, S.; Gómez-Pinillos, A.; del Val Toledo Lobo, M. Prognostic significance of the expression of vascular endothelial growth factors A, B, C, and D and their receptors $\mathrm{R} 1, \mathrm{R} 2$, and $\mathrm{R} 3$ in patients with nonsmall cell lung cancer. Cancer, 2009, 115, 1701-12.

(c) Atif Ali Ahmed; Licensee Bentham Open.

This is an open access article licensed under the terms of the Creative Commons Attribution Non-Commercial License (http://creativecommons.org/licenses/by-nc/3.0/) which permits unrestricted, non-commercial use, distribution and reproduction in any medium, provided the work is properly cited. 